DOI: $10.15393 /$ j3.art.2020.6332

UDC 517.98, 517.521

R. Zarghami Farfar, V. Sadri, R. Ahmadi

\title{
SOME IDENTITIES AND INEQUALITIES FOR G-FUSION FRAMES
}

\begin{abstract}
G-fusion frames, which are obtained from the combination of g-frames and fusion frames, were recently introduced for Hilbert spaces. In this paper, we present a new identity for gframes, which was given by Najati for a special case. Also, by using the idea of this identity and the dual frames, some equalities and inequalities are presented for g-fusion frames.
\end{abstract}

Key words: g-frame, dual g-frame, g-fusion frame, dual g-fusion frame

2010 Mathematical Subject Classification: Primary 42C15;

Secondary 46C99, $41 A 58$.

1. Introduction. Recent developments in the frame theory and their applications are the result of some mathematicians' efforts in this topic (see [10], [13], [12], [3], [6], [8]). By more than half a century, this theory has got interesting applications in different branches of science, such as the filter bank theory, signal and image processing, wireless communications, atomic systems, and the Kadison-Singer problem. In 2005, Balan, Casazza, and others found some useful identities for frames by studying properties of the Parseval frames [2]. Simil ar results for fusion frames, g-frames, and $K$-frames are presented in [18], [21], [1]. In [22], a special kind of frames called g-fusion frames is introduced; they are combinations of g-frames and fusion frames. We present some identities for these frames.

2. Preliminaries. Throughout this paper, $H$ and $K$ are separable Hilbert spaces, $\pi_{V}$ is the orthogonal projection from $H$ onto a closed subspace $V \subset H$, and $\mathcal{B}(H, K)$ is the collection of all the bounded linear operators of $H$ into $K$. If $K=H$, then $\mathcal{B}(H, H)$ will be denoted by $\mathcal{B}(H)$. Also, $\left\{H_{j}\right\}_{j \in \mathbb{J}}$ is a sequence of Hilbert spaces and $\Lambda_{j} \in \mathcal{B}\left(H, H_{j}\right)$ for each

(C) Petrozavodsk State University, 2020 
$j \in \mathbb{J}$, where $\mathbb{J}$ is a subset of $\mathbb{Z}$. The following lemmas from the operator theory will be needed.

Lemma 1. [13] Let $V \subseteq H$ be a closed subspace, and $T$ be a linear bounded operator on $H$. Then

$$
\pi_{V} T^{*}=\pi_{V} T^{*} \pi_{\overline{T V}}
$$

Lemma 2. [21] Let $u \in \mathcal{B}(H)$ be adjoint and $v:=a u^{2}+b u+c$ where $a, b, c \in \mathbb{R}$.

(I) If $a>0$, then

$$
\inf _{\|f\|=1}\langle v f, f\rangle \geq \frac{4 a c-b^{2}}{4 a}
$$

(II) If $a<0$, then

$$
\sup _{\|f\|=1}\langle v f, f\rangle \leq \frac{4 a c-b^{2}}{4 a} .
$$

Lemma 3. [2] If $u, v$ are operators on $H$ satisfying $u+v=i d_{H}$, then $u-v=u^{2}-v^{2}$.

We define the space $\mathscr{H}_{2}:=\left(\sum_{j \in J} \oplus H_{j}\right)_{\ell_{2}}$ by

$$
\mathscr{H}_{2}=\left\{\left\{f_{j}\right\}_{j \in \mathbb{I}}: f_{j} \in H_{j}, \sum_{j \in \mathbb{J}}\left\|f_{j}\right\|^{2}<\infty\right\},
$$

with the inner product defined by

$$
\left\langle\left\{f_{j}\right\},\left\{g_{j}\right\}\right\rangle=\sum_{j \in \mathbb{J}}\left\langle f_{j}, g_{j}\right\rangle .
$$

It is clear that $\mathscr{H}_{2}$ is a Hilbert space with pointwise operations.

Definition 1. [23] We call the sequence $\left\{\Lambda_{j}\right\}_{j \in \mathbb{J}}$ a g-frame for $H$ with respect to $\left\{H_{j}\right\}_{j \in \mathbb{J}}$ if there exist $0<A \leq B<\infty$, such that for each $f \in H$

$$
A\|f\|^{2} \leq \sum_{j \in \mathbb{J}}\left\|\Lambda_{j} f\right\|^{2} \leq B\|f\|^{2}
$$

If $A=B=1$, we call $\left\{\Lambda_{j}\right\}_{j \in \mathbb{J}}$ a Parseval g-frame. The synthesis and analysis operators in g-frames are defined by

$$
T: \mathscr{H}_{2} \longrightarrow H, \quad T^{*}: H \longrightarrow \mathscr{H}_{2}
$$




$$
T\left(\left\{f_{j}\right\}_{j \in \mathbb{I}}\right)=\sum_{j \in \mathbb{J}} \Lambda_{j}^{*} f_{j}, \quad T^{*}(f)=\left\{\Lambda_{j} f\right\}_{j \in \mathbb{J}} .
$$

Therefore, the g-frame operator is defined by

$$
S f=T T^{*} f=\sum_{j \in \mathbb{J}} \Lambda_{j}^{*} \Lambda_{j} f .
$$

The operator $S$ is bounded, positive, and invertible. If $\tilde{\Lambda}_{j}:=\Lambda_{j} S^{-1}$, then $\left\{\tilde{\Lambda}_{j}\right\}_{j \in \mathbb{J}}$ is called a (canonical) dual g-frame of $\left\{\Lambda_{j}\right\}_{j \in \mathbb{J}}$, and we can write

$$
f=\sum_{j \in \mathbb{J}} \tilde{\Lambda}_{j}^{*} \Lambda_{j} f=\sum_{j \in \mathbb{J}} \Lambda_{j}^{*} \tilde{\Lambda}_{j} f .
$$

If $\left\{\Lambda_{j}\right\}_{j \in \mathbb{J}}$ is a g-frame for $H$ with bounds $A$ and $B$, respectively, then $\left\{\tilde{\Lambda}_{j}\right\}_{j \in \mathbb{J}}$ is also a g-frame for $H$ with bounds $B^{-1}$ and $A^{-1}$, respectively.

Definition 2. [22] Let $W=\left\{W_{j}\right\}_{j \in \mathbb{J}}$ be a family of closed subspaces of $H,\left\{v_{j}\right\}_{j \in \mathbb{J}}$ be a family of weights, i. e., $v_{j}>0$. We say $\Lambda:=\left(W_{j}, \Lambda_{j}, v_{j}\right)$ is a g-fusion frame for $H$ if there exist $0<A \leq B<\infty$, such that for each $f \in H$

$$
A\|f\|^{2} \leq \sum_{j \in \mathbb{J}} v_{j}^{2}\left\|\Lambda_{j} \pi_{W_{j}} f\right\|^{2} \leq B\|f\|^{2} .
$$

It is easy to see that these frames are extensions of g-frames. We call $\Lambda$ a Parseval g-fusion frame if $A=B=1$. When the right-hand part of (3) holds, $\Lambda$ is called a g-fusion Bessel sequence for $H$ with the bound $B$. Throughout this paper, $\Lambda$ is a triple $\left(W_{j}, \Lambda_{j}, v_{j}\right)$ with $j \in \mathbb{J}$.

The synthesis and analysis operators in the g-fusion frames are defined by (for more details, we refer [22])

$$
\begin{gathered}
T_{\Lambda}: \mathscr{H}_{2} \longrightarrow H, \quad T_{\Lambda}^{*}: H \longrightarrow \mathscr{H}_{2} \\
T_{\Lambda}\left(\left\{f_{j}\right\}_{j \in \mathbb{J}}\right)=\sum_{j \in \mathbb{J}} v_{j} \pi_{W_{j}} \Lambda_{j}^{*} f_{j}, \quad T_{\Lambda}^{*}(f)=\left\{v_{j} \Lambda_{j} \pi_{W_{j}} f\right\}_{j \in \mathbb{J}} .
\end{gathered}
$$

Thus, the g-fusion frame operator is given by

$$
S_{\Lambda} f=T_{\Lambda} T_{\Lambda}^{*} f=\sum_{j \in \mathbb{J}} v_{j}^{2} \pi_{W_{j}} \Lambda_{j}^{*} \Lambda_{j} \pi_{W_{j}} f .
$$

Therefore,

$$
A i d_{H} \leq S_{\Lambda} \leq B i d_{H}
$$


This means that $S_{\Lambda}$ is a bounded, positive, and invertible operator (with an adjoint inverse), and we have

$$
B^{-1} i d_{H} \leq S_{\Lambda}^{-1} \leq A^{-1} i d_{H}
$$

So, we have the following reconstruction formula for any $f \in H$ :

$$
f=\sum_{j \in \mathbb{J}} v_{j}^{2} \pi_{W_{j}} \Lambda_{j}^{*} \Lambda_{j} \pi_{W_{j}} S_{\Lambda}^{-1} f=\sum_{j \in \mathbb{J}} v_{j}^{2} S_{\Lambda}^{-1} \pi_{W_{j}} \Lambda_{j}^{*} \Lambda_{j} \pi_{W_{j}} f .
$$

Let $\tilde{\Lambda}:=\left(S_{\Lambda}^{-1} W_{j}, \Lambda_{j} \pi_{W_{j}} S_{\Lambda}^{-1}, v_{j}\right)$. Then $\tilde{\Lambda}$ is called the (canonical) dual $g$-fusion frame of $\Lambda$. Hence, for each $f \in H$ we get

$$
f=\sum_{j \in \mathbb{I}} v_{j}^{2} \pi_{W_{j}} \Lambda_{j}^{*} \tilde{\Lambda}_{j} \pi_{\tilde{W}_{j}} f=\sum_{j \in \mathbb{I}} v_{j}^{2} \pi_{W_{j}} \tilde{\Lambda}_{j}^{*} \Lambda_{j} \pi_{W_{j}} f
$$

where $\tilde{W}_{j}:=S_{\Lambda}^{-1} W_{j}, \tilde{\Lambda}_{j}:=\Lambda_{j} \pi_{W_{j}} S_{\Lambda}^{-1}$. Thus, we obtain

$$
\left\langle S_{\Lambda}^{-1} f, f\right\rangle=\sum_{j \in \mathbb{I}} v_{j}^{2}\left\|\tilde{\Lambda}_{j} \pi_{\tilde{W}_{j}} f\right\|^{2} .
$$

3. The Main Results. Let $\left\{\Lambda_{j}\right\}_{j \in \mathbb{J}}$ be a g-frame for $H$ with respect to $\left\{H_{j}\right\}_{j \in \mathbb{J}}$ with bounds $A, B$ and $\left\{\tilde{\Lambda}_{j}\right\}_{j \in \mathbb{J}}$ be a (canonical) dual g-frame of $\left\{\Lambda_{j}\right\}_{j \in \mathbb{J}}$. Suppose that $\mathbb{I} \subseteq \mathbb{J}$ and let

$$
\begin{gathered}
S_{\mathbb{I}}: H \rightarrow H \\
S_{\mathbb{I}} f:=\sum_{j \in \mathbb{I}} \Lambda_{j}^{*} \tilde{\Lambda}_{j} f .
\end{gathered}
$$

This is a general case of the operator $S_{J}$ presented in [21]. We have

$$
\begin{aligned}
\left\|S_{\mathbb{I}} f\right\|^{2}=\left(\sup _{\|h\|=1}\left|\left\langle S_{\mathbb{I}} f, h\right\rangle\right|\right)^{2} & =\sup _{\|h\|=1}\left(\sum_{j}\left|\left\langle\tilde{\Lambda}_{j} f, \Lambda_{j} h\right\rangle\right|\right)^{2} \leq \\
\leq & \sum_{j}\left\|\tilde{\Lambda}_{j} f\right\|^{2} \times \sup _{\|h\|=1} \sum_{j}\left\|\Lambda_{j} h\right\|^{2} \leq B A^{-1}\|f\|^{2} .
\end{aligned}
$$

Thus, $S_{\mathbb{I}} \in \mathcal{B}(H)$ and is positive. From (2) we obtain that $S_{\mathbb{I}}+S_{\mathbb{I}^{c}}=i d_{H}$.

Theorem 1. For $f \in H$, we have

$$
\sum_{j \in \mathbb{I}}\left\langle\tilde{\Lambda}_{j} f, \Lambda_{j} f\right\rangle-\left\|S_{\mathbb{I}} f\right\|^{2}=\sum_{j \in \mathbb{I}^{c}} \overline{\left\langle\tilde{\Lambda}_{j} f, \Lambda_{j} f\right\rangle}-\left\|S_{\mathbb{I}^{c}} f\right\|^{2}
$$


where $\mathbb{I}^{c}$ is the complement of $\mathbb{I}$.

Proof. For each $f \in H$, we have

$$
\begin{aligned}
& \sum_{j \in \mathbb{I}}\left\langle\tilde{\Lambda}_{j} f, \Lambda_{j} f\right\rangle-\left\|\sum_{j \in \mathbb{I}} \Lambda_{j}^{*} \tilde{\Lambda}_{j} f\right\|^{2}=\left\langle S_{\mathbb{I}} f, f\right\rangle-\left\|S_{\mathbb{I}} f\right\|^{2}= \\
& =\left\langle S_{\mathbb{I}} f, f\right\rangle-\left\langle S_{\mathbb{I}}^{*} S_{\mathbb{I}} f, f\right\rangle=\left\langle\left(i d_{H}-S_{\mathbb{I}}\right)^{*} S_{\mathbb{I}} f, f\right\rangle=\left\langle S_{\mathbb{I}^{c}}^{*}\left(i d_{H}-S_{\mathbb{I}^{c}}\right) f, f\right\rangle= \\
& \quad=\left\langle S_{\mathbb{I}^{c}}^{*} f, f\right\rangle-\left\langle S_{\mathbb{I}^{c}}^{*} S_{\mathbb{I}^{c}} f, f\right\rangle=\left\langle f, S_{\mathbb{I}^{c}} f\right\rangle-\left\langle S_{\mathbb{I}^{c}} f, S_{\mathbb{I}^{c}} f\right\rangle= \\
& \quad=\sum_{j \in \mathbb{I}^{c}}\left\langle\Lambda_{j} f, \tilde{\Lambda}_{j} f\right\rangle-\left\|\sum_{j \in \mathbb{I}^{c}} \Lambda_{j}^{*} \tilde{\Lambda}_{j} f\right\|^{2}=\sum_{j \in \mathbb{I}^{c}} \overline{\left\langle\tilde{\Lambda}_{j} f, \Lambda_{j} f\right\rangle}-\left\|\sum_{j \in \mathbb{I}^{c}} \Lambda_{j}^{*} \tilde{\Lambda}_{j} f\right\|^{2}
\end{aligned}
$$

and the proof is complete.

Now, if $\left\{\Lambda_{j}\right\}_{j \in \mathbb{J}}$ is a Parseval g-frame, then $\tilde{\Lambda_{j}}=\Lambda_{j}$, and we obtain the following famous formula presented in [21]:

$$
\sum_{j \in \mathbb{I}}\left\|\Lambda_{j} f\right\|^{2}-\left\|S_{\mathbb{I}} f\right\|^{2}=\sum_{j \in \mathbb{I}^{c}}\left\|\Lambda_{j} f\right\|^{2}-\left\|S_{\mathbb{I}^{c}} f\right\|^{2}
$$

where $S_{\mathbb{I}} f=\sum_{j \in \mathbb{I}} \Lambda_{j}^{*} \Lambda_{j} f$.

The same can be obtained for g-fusion frames. Let $\Lambda$ be a g-fusion frame for $H$ with a (canonical) dual g-fusion frame $\tilde{\Lambda}=\left(\tilde{W}_{j}, \tilde{\Lambda}_{j}, v_{j}\right)$, where $\tilde{W}_{j}=S_{\Lambda} W_{j}$ and $\tilde{\Lambda}_{j}=\Lambda_{j} \pi_{W_{j}} S_{\Lambda}^{-1}$. For simplicity, we denote the following operator with the same symbol $S_{\mathbb{I}}$, where, again, $\mathbb{I}$ is a finite subset of $\mathbb{J}$ :

$$
S_{\mathbb{I}} f=\sum_{j \in \mathbb{I}} v_{j}^{2} \pi_{W_{j}} \Lambda_{j}^{*} \tilde{\Lambda}_{j} \pi_{\tilde{W}_{j}} f, \quad(\forall f \in H) .
$$

It is easy to check that $S_{\mathbb{I}} \in \mathcal{B}(H)$ and positive. Again, we have

$$
S_{\mathbb{I}}+S_{\mathbb{I} c}=i d_{H}
$$

Remark 1. Let $\Lambda$ be a Parseval g-fusion frame for $H$. Since $\mathcal{B}(H)$ is a $C^{*}$-algebra and $S_{\mathbb{I}}$ is positive, so $r\left(S_{\mathbb{I}}\right)=\left\|S_{\mathbb{I}}\right\|$, where $r$ is the spectral radius. Thus

$$
\max _{\lambda \in \sigma\left(S_{\mathbb{I}}\right)}|\lambda|=r\left(S_{\mathbb{I}}\right) \leq 1
$$

and we conclude that $\sigma\left(S_{\mathbb{I}}\right) \in[0,1]$.

Theorem 2. Let $f \in H$; then

$$
\sum_{j \in \mathbb{I}} v_{j}^{2}\left\langle\tilde{\Lambda}_{j} \pi_{\tilde{W}_{j}} f, \Lambda_{j} \pi_{W_{j}} f\right\rangle-\left\|S_{\mathbb{I}} f\right\|^{2}=\sum_{j \in \mathbb{I}^{c}} v_{j}^{2} \overline{\left\langle\tilde{\Lambda}_{j} \pi_{\tilde{W}_{j}} f, \Lambda_{j} \pi_{W_{j}} f\right\rangle}-\left\|S_{\mathbb{I}} f\right\|^{2} .
$$


Proof. The proof follows a similar argument as in the proof of Theorem 1 .

Corollary 1. Let $\Lambda$ be a Parseval g-fusion frame for $H$. Then

$$
\begin{aligned}
& \sum_{j \in \mathbb{I}} v_{j}^{2}\left\|\Lambda_{j} \pi_{W_{j}} f\right\|^{2}-\| \sum_{j \in \mathbb{I}} v_{j}^{2} \pi_{W_{j}} \Lambda_{j}^{*} \Lambda_{j} \pi_{W_{j}} f \|^{2}= \\
&=\sum_{j \in \mathbb{I}^{c}} v_{j}^{2}\left\|\Lambda_{j} \pi_{W_{j}} f\right\|^{2}-\left\|\sum_{j \in \mathbb{I}^{c}} v_{j}^{2} \pi_{W_{j}} \Lambda_{j}^{*} \Lambda_{j} \pi_{W_{j}} f\right\|^{2} .
\end{aligned}
$$

Moreover,

$$
\sum_{j \in \mathbb{I}} v_{j}^{2}\left\|\Lambda_{j} \pi_{W_{j}} f\right\|^{2}+\left\|\sum_{j \in \mathbb{I}^{c}} v_{j}^{2} \pi_{W_{j}} \Lambda_{j}^{*} \Lambda_{j} \pi_{W_{j}} f\right\|^{2} \geq \frac{3}{4}\|f\|^{2} .
$$

Proof. If $f \in H$, we obtain

$$
\begin{aligned}
\sum_{j \in \mathbb{I}} v_{j}^{2}\left\|\Lambda_{j} \pi_{W_{j}} f\right\|^{2}+\left\|S_{\mathbb{I}^{c}} f\right\|^{2}=\left\langle\left(S_{\mathbb{I}}+S_{\mathbb{I}^{c}}^{2}\right) f, f\right\rangle & = \\
=\left\langle\left(S_{\mathbb{I}}+i d_{H}-2 S_{\mathbb{I}}+S_{\mathbb{I}}^{2}\right) f, f\right\rangle & =\left\langle\left(i d_{H}-S_{\mathbb{I}}+S_{\mathbb{I}}^{2}\right) f, f\right\rangle .
\end{aligned}
$$

Now, by Lemma 2 for $a=1, b=-1$, and $c=1$, the inequality holds.

Corollary 2. Let $\Lambda$ be a Parseval g-fusion frame for $H$. Then

$$
0 \leq S_{\mathbb{I}}-S_{\mathbb{I}}^{2} \leq \frac{1}{4} i d_{H}
$$

Proof. We have $S_{\mathbb{I}} S_{\mathbb{I} c}=S_{\mathbb{I}} S_{\mathbb{I}}$. Then $0 \leq S_{\mathbb{I}} S_{\mathbb{I} c}=S_{\mathbb{I}}-S_{\mathbb{I}}^{2}$. Also, by Lemma 2, we get

$$
S_{\mathbb{I}}-S_{\mathbb{I}}^{2} \leq \frac{1}{4} i d_{H}
$$

The proof is complete.

Theorem 3. Let $\Lambda$ be a g-fusion frame with the $g$-fusion frame operator $S_{\Lambda}$. If $\mathbb{I} \subseteq \mathbb{J}$ and $f \in H$, then

$$
\sum_{j \in \mathbb{I}} v_{j}^{2}\left\|\Lambda_{j} \pi_{W_{j}} f\right\|^{2}+\left\|S_{\Lambda}^{-\frac{1}{2}} S_{\mathbb{I}^{c}} f\right\|^{2}=\sum_{j \in \mathbb{I}^{c}} v_{j}^{2}\left\|\Lambda_{j} \pi_{W_{j}} f\right\|^{2}+\left\|S_{\Lambda}^{-\frac{1}{2}} S_{\mathbb{I}} f\right\|^{2} .
$$

Proof. Let $\Theta_{j}:=\Lambda_{j} \pi_{W_{j}} S_{\Lambda}^{-\frac{1}{2}}$ and $X_{j}:=S_{\Lambda}^{-\frac{1}{2}} W_{j}$. Example 2.2 [22] shows that $\left(X_{j}, \Theta_{j}, v_{j}\right)$ is a Parseval g-fusion frame for $H$. Then, by Corollary 1 , we have 


$$
\begin{aligned}
& \sum_{j \in \mathbb{I}} v_{j}^{2}\left\|\Theta_{j} \pi_{X_{j}} f\right\|^{2}-\| \sum_{j \in \mathbb{I}} v_{j}^{2} \pi_{X_{j}} \Theta_{j}^{*} \Theta_{j} \pi_{X_{j}} f \|^{2}= \\
&=\sum_{j \in \mathbb{I}^{c}} v_{j}^{2}\left\|\Theta_{j} \pi_{X_{j}} f\right\|^{2}-\left\|\sum_{j \in \mathbb{I}^{c}} v_{j}^{2} \pi_{X_{j}} \Theta_{j}^{*} \Theta_{j} \pi_{X_{j}} f\right\|^{2} .
\end{aligned}
$$

By replacing $f$ by $S_{\Lambda}^{\frac{1}{2}} f$ and the fact that

$$
\begin{aligned}
\sum_{j \in \mathbb{I}} v_{j}^{2} \pi_{X_{j}} \Theta_{j}^{*} \Theta_{j} \pi_{X_{j}} f & =\sum_{j \in \mathbb{I}} v_{j}^{2}\left(\Theta_{j} \pi_{X_{j}}\right)^{*} \Theta_{j} \pi_{X_{j}} f= \\
& =\sum_{j \in \mathbb{I}} v_{j}^{2}\left(\Lambda_{j} \pi_{W_{j}} S_{\Lambda}^{-\frac{1}{2}} \pi_{X_{j}}\right)^{*} \Lambda_{j} \pi_{W_{j}} S_{\Lambda}^{-\frac{1}{2}} \pi_{X_{j}} f= \\
& =\sum_{j \in \mathbb{I}} v_{j}^{2} S_{\Lambda}^{-\frac{1}{2}} \pi_{W_{j}} \Lambda_{j}^{*} \Lambda_{j} \pi_{W_{j}} S_{\Lambda}^{-\frac{1}{2}} f= \\
& =S_{\Lambda}^{-\frac{1}{2}} S_{\mathbb{I}} S_{\Lambda}^{-\frac{1}{2}} f,
\end{aligned}
$$

the proof is complete.

Corollary 1. Let $\Lambda$ be a g-fusion frame with the $g$-fusion frame operator $S_{\Lambda}$. If $\mathbb{I} \subseteq \mathbb{J}$, then

$$
0 \leq S_{\mathbb{I}}-S_{\mathbb{I}} S_{\Lambda}^{-1} S_{\mathbb{I}} \leq \frac{1}{4} S_{\Lambda}
$$

Proof. In the proof of Theorem 3, we showed that

$$
\sum_{j \in \mathbb{I}} v_{j}^{2} \pi_{X_{j}} \Theta_{j}^{*} \Theta_{j} \pi_{X_{j}} f=S_{\Lambda}^{-\frac{1}{2}} S_{\mathbb{I}} S_{\Lambda}^{-\frac{1}{2}} f .
$$

By Corollary 2, we get

$$
0 \leq \sum_{j \in \mathbb{I}} v_{j}^{2} \pi_{X_{j}} \Theta_{j}^{*} \Theta_{j} \pi_{X_{j}} f-\left(\sum_{j \in \mathbb{I}} v_{j}^{2} \pi_{X_{j}} \Theta_{j}^{*} \Theta_{j} \pi_{X_{j}} f\right)^{2} \leq \frac{1}{4} i d_{H} .
$$

Therefore,

$$
0 \leq S_{\Lambda}^{-\frac{1}{2}}\left(S_{\mathbb{I}}-S_{\mathbb{I}} S_{\Lambda}^{-1} S_{\mathbb{I}}\right) S_{\Lambda}^{-\frac{1}{2}} \leq \frac{1}{4} i d_{H}
$$

and the proof is complete.

Corollary 2. Suppose that $\Lambda$ is a $g$-fusion frame with the g-fusion frame operator $S_{\Lambda}$. If $\mathbb{I} \subseteq \mathbb{J}$ and $f \in H$, then

$$
\sum_{j \in \mathbb{I}} v_{j}^{2}\left\|\Lambda_{j} \pi_{W_{j}} f\right\|^{2}+\left\|S_{\Lambda}^{-\frac{1}{2}} S_{\mathbb{I}^{c}} f\right\|^{2} \geq \frac{3}{4}\left\|S_{\Lambda}^{-1}\right\|^{-1}\|f\|^{2} .
$$


Proof. By Theorem 3 and Corollary 1, we can write

$$
\begin{aligned}
& \sum_{j \in \mathbb{I}} v_{j}^{2}\left\|\Lambda_{j} \pi_{W_{j}} f\right\|^{2}+\left\|S_{\Lambda}^{-\frac{1}{2}} S_{\mathbb{I}^{c}} f\right\| \\
&=\sum_{j \in \mathbb{I}} v_{j}^{2}\left\|\Theta_{j} \pi_{X_{j}} S_{\Lambda}^{\frac{1}{2}} f\right\|^{2}+\left\|\sum_{j \in \mathbb{I}^{c}} v_{j}^{2} \pi_{X_{j}} \Theta_{j}^{*} \Theta_{j} \pi_{X_{j}} S_{\Lambda}^{\frac{1}{2}} f\right\|^{2} \geq \\
& \quad \geq \frac{3}{4}\left\|S_{\Lambda}^{\frac{1}{2}} f\right\|^{2}=\frac{3}{4}\left\langle S_{\Lambda} f, f\right\rangle \geq \frac{3}{4}\left\|S_{\Lambda}^{-1}\right\|^{-1}\|f\|^{2} .
\end{aligned}
$$

The poof is complete.

Theorem 4. Let $\Lambda$ be a Parseval g-fusion frame for $H$ and $\mathbb{I} \subseteq \mathbb{J}$. Then

$$
\begin{aligned}
& \text { (I) } 0 \leq S_{\mathbb{I}}-S_{\mathbb{I}}^{2} \leq \frac{1}{4} i d_{H} . \\
& \text { (II) } \frac{1}{2} i d_{H} \leq S_{\mathbb{I}}^{2}+S_{\mathbb{I}^{c}}^{2} \leq \frac{3}{2} i d_{H} .
\end{aligned}
$$

Proof. (I) Since $S_{\mathbb{I}}+S_{\mathbb{I}^{c}}=i d_{H}, S_{\mathbb{I}} S_{\mathbb{I}^{c}}+S_{\mathbb{I}^{c}}^{2}=S_{\mathbb{I}^{c}}$. Thus,

$$
S_{\mathbb{I}} S_{\mathbb{I}^{c}}=S_{\mathbb{I}^{c}}-S_{\mathbb{I}^{c}}^{2}=S_{\mathbb{I}^{c}}\left(i d_{H}-S_{\mathbb{I}^{c}}\right)=S_{\mathbb{I}^{c}} S_{\mathbb{I}}
$$

But $\Lambda$ is Parseval, so $0 \leq S_{\mathbb{I}} S_{\mathbb{I}^{c}}=S_{\mathbb{I}}-S_{\mathbb{I}}^{2}$. On the other hand, by Lemma 3, we get

$$
S_{\mathbb{I}}-S_{\mathbb{I}}^{2} \leq \frac{1}{4} i d_{H}
$$

(II) We have seen that $S_{\mathbb{I}} S_{\mathbb{I}^{c}}=S_{\mathbb{I}^{c}} S_{\mathbb{I}}$; then, by Lemma 3,

$$
S_{\mathbb{I}}^{2}+S_{\mathbb{I}^{c}}^{2}=i d_{H}-2 S_{\mathbb{I}} S_{\mathbb{I}^{c}}=2 S_{\mathbb{I}}^{2}-2 S_{\mathbb{I}}+i d_{H} \geq \frac{1}{2} i d_{H} .
$$

On the other hand, we have, again, by Lemma 3 and $0 \leq S_{\mathbb{I}}-S_{\mathbb{I}}^{2}$ :

$$
S_{\mathbb{I}}^{2}+S_{\mathbb{I} c}^{2} \leq i d_{H}+2 S_{\mathbb{I}}-2 S_{\mathbb{I}}^{2} \leq \frac{3}{2} i d_{H}
$$

This completes the proof.

Corollary 1. Let $\Lambda$ be a g-fusion frame with the g-fusion frame operator $S_{\Lambda}$. If $\mathbb{I} \subseteq \mathbb{J}$, then

$$
\frac{1}{2} S_{\Lambda} \leq S_{\mathbb{I}} S_{\Lambda}^{-1} S_{\mathbb{I}}-S_{\mathbb{I}^{c}} S_{\Lambda}^{-1} S_{\mathbb{I}^{c}} \leq \frac{3}{2} S_{\Lambda}
$$


Proof. We have

$$
\sum_{j \in \mathbb{I}} v_{j}^{2} \pi_{X_{j}} \Theta_{j}^{*} \Theta_{j} \pi_{X_{j}} f=S_{\Lambda}^{-\frac{1}{2}} S_{\mathbb{I}} S_{\Lambda}^{-\frac{1}{2}} f .
$$

Therefore, similarly to the proof of Corollary 1, we get, by Theorem 4, item (II),

$$
\frac{1}{2} i d_{H} \leq\left(S_{\Lambda}^{-\frac{1}{2}} S_{\mathbb{I}} S_{\Lambda}^{-\frac{1}{2}}\right)^{2}+\left(S_{\Lambda}^{-\frac{1}{2}} S_{\mathbb{I}} S_{\Lambda}^{-\frac{1}{2}}\right)^{2} \leq \frac{3}{2} i d_{H},
$$

and the proof is now evident.

Theorem 5. Let $\Lambda$ be a g-fusion frame with the g-fusion frame operator $S_{\Lambda}$. If $\mathbb{I} \subseteq \mathbb{J}$, then, for any $f \in H$,

$$
\begin{aligned}
\sum_{j \in \mathbb{I}} v_{j}^{2}\left\|\Lambda_{j} \pi_{W_{j}} f\right\|^{2}-\sum_{j \in \mathbb{J}} v_{j}^{2}\left\|\tilde{\Lambda}_{j} \pi_{\tilde{W}_{j}} M_{\mathbb{I}} f\right\|^{2}= \\
=\sum_{j \in \mathbb{I}^{c}} v_{j}^{2}\left\|\Lambda_{j} \pi_{W_{j}} f\right\|^{2}-\sum_{j \in \mathbb{J}} v_{j}^{2}\left\|\tilde{\Lambda}_{j} \pi_{\tilde{W}_{j}} M_{\mathbb{I}^{c}} f\right\|^{2}
\end{aligned}
$$

where

$$
M_{\mathbb{I}} f=\sum_{j \in \mathbb{I}} v_{j}^{2} \pi_{W_{j}} \Lambda_{j}^{*} \Lambda_{j} \pi_{\tilde{W}_{j}} f .
$$

Proof. Via the definition of $S_{\Lambda}$, it is clear that $M_{\mathbb{I}}+M_{\mathbb{I} c}=S_{\Lambda}$. Therefore, $S_{\Lambda}^{-1} M_{\mathbb{I}}+S_{\Lambda}^{-1} M_{\mathbb{I}}=i d_{H}$. Hence, by Lemma 3

$$
S_{\Lambda}^{-1} M_{\mathbb{I}}-S_{\Lambda}^{-1} M_{\mathbb{I}}=\left(S_{\Lambda}^{-1} M_{\mathbb{I}}\right)^{2}-\left(S_{\Lambda}^{-1} M_{\mathbb{I}}\right)^{2} .
$$

Thus, for each $f, g \in H$ we obtain

$$
\left\langle S_{\Lambda}^{-1} M_{\mathbb{I}} f, g\right\rangle-\left\langle S_{\Lambda}^{-1} M_{\mathbb{I}} S_{\Lambda}^{-1} M_{\mathbb{I}} f, g\right\rangle=\left\langle S_{\Lambda}^{-1} M_{\mathbb{I}} f, g\right\rangle-\left\langle S_{\Lambda}^{-1} M_{\mathbb{I}} S_{\Lambda}^{-1} M_{\mathbb{I}^{c}} f, g\right\rangle .
$$

We choose $g$ to be $g=S_{\Lambda} f$, and we can get

$$
\left\langle M_{\mathbb{I}} f, f\right\rangle-\left\langle S_{\Lambda}^{-1} M_{\mathbb{I}} f, M_{\mathbb{I}} f\right\rangle=\left\langle M_{\mathbb{I}^{c}} f, f\right\rangle-\left\langle S_{\Lambda}^{-1} M_{\mathbb{I}^{c}} f, M_{\mathbb{I}^{c}} f\right\rangle .
$$

Finally, by (6), the proof is complete. $\square$ 


\section{References}

[1] Arabyani F., Minaei G. M., Anjidani E. On Some Equalities and Inequalities for K-Frames. Indian J. Pure. Appl. Math., 2019, vol. 50 (2), pp. 297308. DOI: https://doi.org/10.1007/s13226-019-0325-8.

[2] Balan R., Casazza P. G., Edidin D., Kutyniok G. A New Identity for Parseval Frames. Proc. Amer. Math. Soc., 2007, vol. 135, pp. 1007-1015.

DOI: https://doi.org/10.1090/S0002-9939-06-08930-1.

[3] Blocsli H., Hlawatsch H. F., Fichtinger H. G. Frame-Theoretic analysis of oversampled filter bank, IEEE Trans. Signal Processing., 1998, vol. 46 (12), pp. 3256-3268.

[4] Candes E. J., Donoho D. L. New tight frames of curvelets and optimal representation of objects with piecewise $C^{2}$ singularities. Comm. Pure and App. Math., 2004, vol. 57 (2), pp. 219 - 266.

DOI: https://doi.org/10.1002/cpa.10116.

[5] Casazza P. G., Christensen O. Perturbation of Operators and Application to Frame Theory. J. Fourier Anal. Appl., 1997, vol. 3, pp. 543-557.

[6] Casazza P. G., Kutyniok G. Frames of Subspaces. Contemp. Math., 1998, vol. 345 , pp. 87-114.

[7] Casazza P. G., Kutyniok G., Li S. Fusion Frames and distributed processing. Appl. comput. Harmon. Anal., 2004, vol. 57 (2), pp. 219-266. 25(1), 2008, 114-132.

[8] Christensen O. An Introduction to Frames and Riesz Bases. Birkhäuser, 2016.

[9] Diestel J. Sequences and series in Banach spaces. Springer-Verlag, New York, 1984.

[10] Duffin R. J., Schaeffer A. C. A class of nonharmonic Fourier series. Trans. Amer. Math. Soc, 1952, vol. 72 (1), pp. 341-366.

[11] Faroughi M. H., Ahmadi R. Some Properties of C-Frames of Subspaces. J. Nonlinear Sci. Appl., 2008, vol. 1 (3), pp. 155-168.

[12] Feichtinger H. G., Werther T. Atomic Systems for Subspaces. Proceedings SampTA. Orlando, FL., 2001, pp. 163-165.

[13] Găvruţa P. On the duality of fusion frames. J. Math. Anal. Appl., 2007, vol. 333, pp. 871-879.

[14] Hansen F., Pečarić J., Perić I. Jensens Operator inequality and its converses. Math. Scand., 2007, vol. 100, pp. 61-73.

[15] Heuser H. Functional Analysis. John Wiley, New York, 1991. 
[16] Kadison R., Singer I. Extensions of pure states. American Journal of Math., 1959, vol. 81, pp. 383-400.

[17] Khayyami M., Nazari A. Construction of Continuous g-Frames and Continuous Fusion Frames. Sahand Comm. Math. Anal., 2016, vol. 4 (1), pp. $43-55$.

[18] Li D., Leng J. On Some New Inequalities for Fusion Frames in Hilbert Spaces. Math. Ineq. Appl., 2017, vol. 20 (3), pp. 889-900.

DOI: https://doi.org/10.7153/mia-20-56.

[19] Matković M., Pečarić J., Perić I. A variant of Jensen's Inequality of Mercer's Type For Operators with Applications. Linear Algebra Appl., 2006, vol. 418, pp. 551-564.

[20] Najati A., Faroughi M. H., Rahimi A. g-frames and stability of g-frames in Hilbert spaces. Methods of Functional Analysis and Topology., 2008, vol. 14 (3), pp. 305-324.

[21] Najati A., H., Rahimi A. Generalized frames in Hilbert spaces. Bull. Iranian Math. Soc., 2009, vol. 35 (1), pp. 97-109.

[22] Sadri V., Rahimlou G., Ahmadi R., Zarghami Farfar R. Construction of $g$-fusion frames in Hilbert spaces. Inf. Dim. Anal. Quan. Prob.(IDA-QP), to appear 2019.

[23] Sun W. G-Frames and G-Riesz bases., J. Math. Anal. Appl., 2006, vol. 326, pp. $437-452$.

Received May 27, 2019.

In revised form, November 29, 2019.

Accepted January 11, 2020.

Published online April 29, 2020.

Ramazan Zarghami Farfar

University of Tabriz

Marand Faculty of Technical and Engineering, Iran.

E-mail: zarghamir@gmail.com

Vahid Sadri

Department of Mathematics, Faculty of Tabriz Branch,

Technical and Vocational University (TVU), East Azarbaijan, Iran.

E-mail: vahidsadri57@gmail.com

Reza Ahmadi

University of Tabriz

Institute of Fundamental Sciences, Iran.

E-mail: rahmadi@tabrizu.ac.ir 